\title{
Photochemical mechanism of an atypical algal phytochrome
}

DOI:

10.1002/cbic.201800016

\section{Document Version}

Accepted author manuscript

Link to publication record in Manchester Research Explorer

\section{Citation for published version (APA):}

Choudry, U., Heyes, D., Hardman, S., Sakuma, M., Sazanovich, I. V., Woodhouse, J., De La Mora, E., Pedersen, M. N., Wulff, M., Weik, M., Schiro, G., \& Scrutton, N. (2018). Photochemical mechanism of an atypical algal phytochrome. ChemBioChem: a European journal of chemical biology , 19(10), 1036-1043. https://doi.org/10.1002/cbic.201800016

\section{Published in:}

ChemBioChem: a European journal of chemical biology

\section{Citing this paper}

Please note that where the full-text provided on Manchester Research Explorer is the Author Accepted Manuscript or Proof version this may differ from the final Published version. If citing, it is advised that you check and use the publisher's definitive version.

\section{General rights}

Copyright and moral rights for the publications made accessible in the Research Explorer are retained by the authors and/or other copyright owners and it is a condition of accessing publications that users recognise and abide by the legal requirements associated with these rights.

\section{Takedown policy}

If you believe that this document breaches copyright please refer to the University of Manchester's Takedown Procedures [http://man.ac.uk/04Y6Bo] or contact uml.scholarlycommunications@manchester.ac.uk providing relevant details, so we can investigate your claim.

\section{OPEN ACCESS}




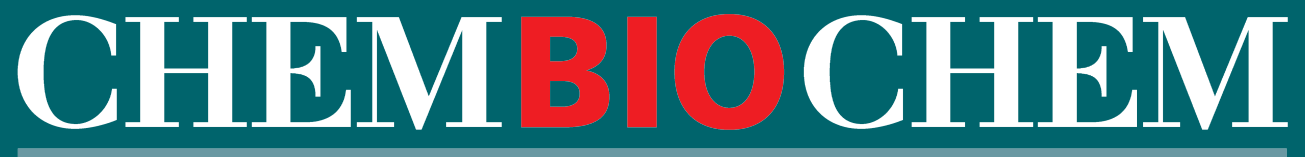

SYNTHETIC BIOLOGY \& BIO-NANOTECHNOLOGY

\section{Accepted Article}

Title: Photochemical mechanism of an atypical algal phytochrome

Authors: Nigel Scrutton, Uzma Choudry, Derren Heyes, Samantha Hardman, Michiyo Sakuma, Igor Sazanovich, Joyce Woodhouse, Eugenio De La Mora, Martin Pederson, Michael Wulff, Martin Weik, and Giorgio Schiro

This manuscript has been accepted after peer review and appears as an Accepted Article online prior to editing, proofing, and formal publication of the final Version of Record (VoR). This work is currently citable by using the Digital Object Identifier (DOI) given below. The VoR will be published online in Early View as soon as possible and may be different to this Accepted Article as a result of editing. Readers should obtain the VoR from the journal website shown below when it is published to ensure accuracy of information. The authors are responsible for the content of this Accepted Article.

To be cited as: ChemBioChem 10.1002/cbic.201800016

Link to VoR: http://dx.doi.org/10.1002/cbic.201800016 


\title{
Photochemical mechanism of an atypical algal phytochrome
}

\author{
Uzma Choudry ${ }^{[a] \dagger}$, Derren J. Heyes ${ }^{[a] \dagger}$, Samantha J. O. Hardman ${ }^{[a] \dagger}{ }^{\dagger}$ Michiyo Sakuma ${ }^{[a]}$, Igor V. \\ Sazanovich $^{[b]}$, Joyce Woodhouse ${ }^{[c]}$, Eugenio De La Mora ${ }^{[c]}$, Martin N. Pedersen ${ }^{[d]}$, Michael Wulff ${ }^{[d]}$, \\ Martin Weik ${ }^{[c]}$, Giorgio Schirò ${ }^{[c]}$, Nigel S. Scrutton* ${ }^{[a]}$
}

\begin{abstract}
Phytochromes are bilin-containing photoreceptors that are typically sensitive to the red / far-red region of the visible spectrum. Recently phytochromes from certain eukaryotic algae have become attractive targets for optogenetic applications because of their unique ability to respond to multiple wavelengths of light. Here, we have used a combination of time-resolved spectroscopy and structural approaches across picosecond to second timescales to map photochemical mechanisms and structural change in this atypical group of phytochromes. The photochemistry of an orange / far-red light sensitive algal phytochrome from Dolihomastix tenuilepis has been investigated using a combination of visible, infra-red and X-ray scattering probes. The entire photocycle, correlated with accompanying structural changes in the cofactor / protein, are reported. Our study identifies a complex photocycle for this atypical phytochrome. It also highlights a need to combine outcomes from a range of biophysical approaches to unravel complex photochemical and macromolecular processes in multi-domain photoreceptor proteins that are the basis of biological light-mediated signalling.
\end{abstract}

\section{Introduction}

The red / far-red light-sensitive phytochromes (Phys) are essential photoreceptors that regulate a myriad of photomorphological processes in plants, bacteria, fungi and algae (e.g. seed germination, leaf-opening). ${ }^{[1,2]}$ Phys contain a covalently bound bilin cofactor and typically photoconvert between a red light-absorbing $P_{R}$ state and a far-red light-absorbing $P_{F R}$ state. ${ }^{[1]}$ Photoconversion between the two states is triggered by an ultrafast $E / Z$ isomerisation of the bilin tetrapyrrole, leading to slower protein structural change (microsecond to millisecond timescales) to form an active signalling conformation. The initial photoisomerisation has been studied in numerous Phys from different organisms using time resolved UV-Vis, infrared (IR) and Raman spectroscopies and is known to involve isomerisation

[a] U. Choudry, Dr.D.J.Heyes, Dr.S.J.O.Hardman, M. Sakuma, Prof. N. S. Scrutton

Manchester Institute of Biotechnology, University of Manchester, 131 Princess Street, Manchester M1 7DN (UK)

E-mail: nigel.scrutton@manchester.ac.uk

[b] Dr. I. V. Sazanovich

Central Laser Facility, Research Complex at Harwell, Science and Technology Facilities Council, Harwell Oxford, Didcot, OX11 OQX (UK)

[c] Dr. G. Schirò, J. Woodhouse, Dr. E. De La Mora, Dr. M. Weik CNRS, Université Grenoble Alpes, CEA - Institut de Biologie Structurale, Grenoble 38044 (France)

[d] Dr. M. N. Pedersen, Dr. M. Wulff

European Synchrotron Radiation Facility, Grenoble 38044 (France)

$\dagger \quad$ These authors contributed equally to this work

Supporting information for this article is given via a link at the end of the document. around the $\mathrm{C} 15$ and $\mathrm{C} 16$ double bond between ring $\mathrm{C}$ and $\mathrm{D}$ of the bilin ${ }^{[1,3-9]}$. X-ray crystallography studies of the photosensory region of a bacterial Phy in conjunction with time-resolved X-ray scattering data have shown that this causes a subsequent refolding of a conserved tongue region leading to large-scale conformational change in the remainder of the protein. ${ }^{[10,11]}$

Although Phys have proven to be attractive targets for new applications in the field of optogenetics ${ }^{[12,13]}$, their use has been limited due to their sensitivity to only a narrow range of wavelengths (red and far-red light). However, it was recently discovered that Phys from certain eukaryotic algae exhibit diverse spectral properties that are capable of spanning most of the visible region. ${ }^{[14]}$ The origin of this spectral diversity is currently unknown and it is unclear whether these algal Phys undergo similar photocycles to those found in typical Phys. In order to exploit fully the potential of algal Phys as new optogenetic tools, it is important to develop an understanding of the photochemistry and photodynamics that underpin biological signalling.

We have investigated in detail the kinetics and structural changes of both the bilin cofactor and the protein throughout the entire photocycle of the photosensory region of an algal Phy by using a combination of time-resolved laser photoexcitation spectroscopy across multiple timescales (continuously from picoseconds to seconds). Spectral changes in the visible region were monitored to report on significant chromophore changes whilst the mid-infra-red spectral region was used to report on finer structural changes in the bilin cofactor. These measurements have been combined with time-resolved small and wide angle X-ray scattering (S/WAXS) measurements to understand how these spectral changes are coupled to larger conformational changes in the protein.

\section{Results and Discussion}

The eukaryotic algal Phy from Dolihomastix tenuilepis (Dten) has a far-red light-absorbing, $P_{F R}$, state, with an absorption maximum at $714 \mathrm{~nm}$ and lower intensity features at 375 and 614 $\mathrm{nm}$, and an orange light-absorbing, $\mathrm{P}_{\mathrm{o}}$, state with an absorption maximum at $598 \mathrm{~nm}$ and a lower intensity feature at $354 \mathrm{~nm}$ (Figure 1). ${ }^{[14]}$ Time-resolved spectroscopy has been used to measure optical changes in the visible and infra-red (IR) regions that occur upon photoconversion of both states of the protein. Measurements in the visible region spanned the complete timerange of the photoreaction (ps-ms). These measurements were repeated in $\mathrm{H}_{2} \mathrm{O}$ and $\mathrm{D}_{2} \mathrm{O}$ buffers to identify any kinetic isotope effects associated with each of the individual steps of the photocycle, and to provide direct comparisons to the IR data, collected on samples in $\mathrm{D}_{2} \mathrm{O}$ buffer. The IR measurements were recorded over the first $400 \mu$ s of the reaction to monitor the initial structural changes of the reaction.

For internal use, please do not delete. Submitted_Manuscript 


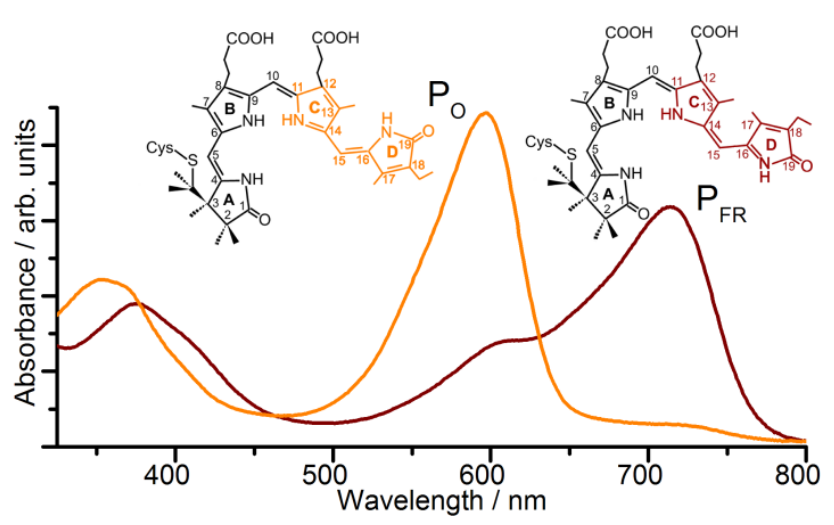

Figure 1. Absorption spectra, and structures of bilin chromophore, of Dten Phy in the orange-absorbing state and the far-red-absorbing states.

Global analysis has been used to model the time-resolved absorption difference spectra from the visible and IR measurements by fitting the data to a sequential, unbranched model to yield evolution associated difference spectra (EADS). Previous studies of similar photoreactions suggest that it is unlikely that any significant reaction branching will occur and that a simple sequential reaction scheme will fit the data adequately. ${ }^{[15,16]}$ Major features in the IR spectral region monitored here include the stretches of the $\mathrm{C}=\mathrm{O}$ bonds on rings $A$ and $D$ at $\sim 1730$ and $\sim 1720 \mathrm{~cm}^{-1}$, respectively and $C=C$ stretches at $\sim 1595,1620,1627$, and $1648 \mathrm{~cm}^{-1}$, which represent the bonds between rings $B$ and $C, A$ and $B, C$ and $D$, and the vinyl group attached to ring $D$, respectively. ${ }^{[17]}$ Features from the protein amide I group and amino acid side chains occur at $\sim 1650$ and $\sim 1450 \mathrm{~cm}^{-1}$. ${ }^{[18]}$ Contour maps of the raw data along with the corresponding EADSs are shown in the main manuscript (Figures 2 and 3); more extensive data and analysis figures can be found in the supporting information (Figures S1-6). The photoconversion of the $\mathrm{Po}_{\mathrm{O}}$ state gives rise to three resolvable steps in the visible region (represented by EADS 1-4 in Figure 2). There is an initial bleach of the ground state absorption feature together with the appearance of a new absorption peak at $670 \mathrm{~nm}$ and broad excited state absorption features between 400 and $500 \mathrm{~nm}$ (Figure 2B,D, EADS1). EADS1 then evolves into EADS2, which has an absorbance maximum at $660 \mathrm{~nm}$ and a proportionally similar sized excited state absorption band, with a lifetime of 116 ps (112 ps in $\mathrm{D}_{2} \mathrm{O}$ ).
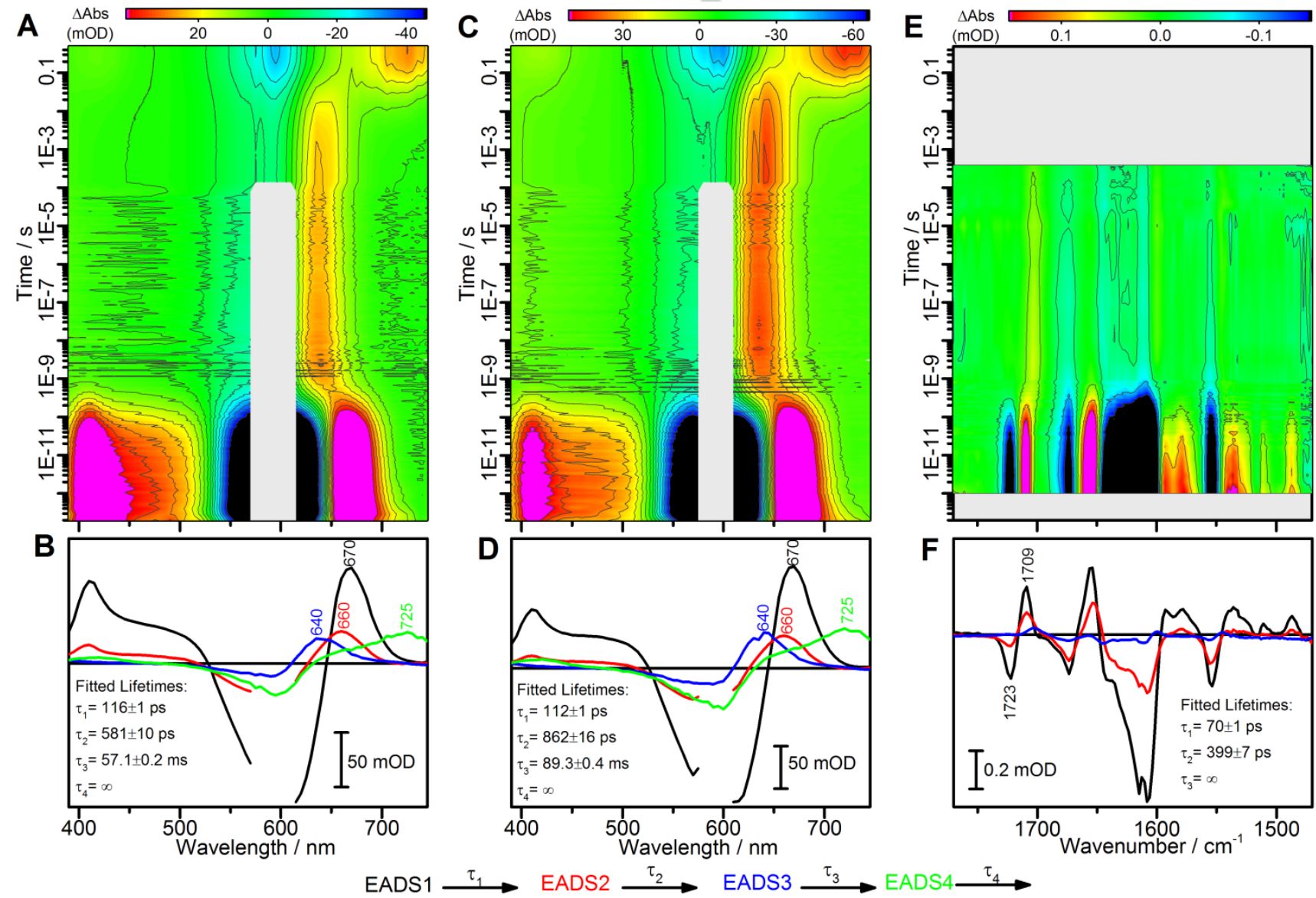

Figure 2. Contour plots of the raw data and evolution associated difference spectra (EADS) resulting from a global analysis of time-resolved visible and IR transient absorption data, collected after excitation of the $\mathrm{P}_{\mathrm{o}}$ state. $\mathbf{A}$ and $\mathbf{B}$, visible data collected on Dten Phy in $\mathrm{H}_{2} \mathrm{O}$ buffer; $\mathbf{C}$ and $\mathbf{D}$, visible data collected on Dten Phy in $\mathrm{D}_{2} \mathrm{O}$ buffer;. $\mathbf{E}$ and $\mathbf{F}$, IR data collected on Dten Phy in $\mathrm{D}_{2} \mathrm{O}$ buffer. Visible data were recorded from 0.2 ps until no further spectral changes were observed $(495 \mathrm{~ms})$, IR data were recorded over a 1 ps - $390 \mu \mathrm{s}$ window. The gap in the $580-605$ nm range is due to scattered pump light obscuring the transient absorption signal.

For internal use, please do not delete. Submitted_Manuscript 
In the IR region, although there are differences in the absolute intensities, the shape and positions of the spectral features are very similar between EADS1 and EADS2 (Figure 2F). Hence, it seems unlikely that this transition corresponds to the $E / Z$ bond isomerisation, which would be expected to produce noticeable changes in this spectral region. Instead, this kinetic component is likely to represent excited state relaxation, potentially of the excited state $P_{0}$ which has not been isomerised. The bleach at $1723 \mathrm{~cm}^{-1}$ and transient feature at $1709 \mathrm{~cm}^{-1}$ that is already present in EADS1 suggests a change in the $C=O$ bond on ring $D$ and hence, it is possible that the $E / Z$ isomerisation of the bilin cofactor may have already occurred before the first measurement at 1 ps after excitation. This assignment is supported by the residuals of the analysis of the visible data (Figures S1 and S3) where it is possible to observe a very short lived component that could not be resolved kinetically due to the lack of data points on this timescale, and proximity to the time resolution of the system ( $0.2 \mathrm{ps})$. The next reaction intermediate, which has a visible absorption maximum at 640 $\mathrm{nm}$ (EADS3), is formed with a lifetime of 581 ps in $\mathrm{H}_{2} \mathrm{O}$ (862 ps in $\mathrm{D}_{2} \mathrm{O}$ ). The IR spectra also display significant changes for this step, both in intensity and position of dominant spectral features. There are changes across the full spectral range monitored, with apparently new features observed in regions assigned to $C=C$ stretches of the bilin, as well as amide bands from the protein, these likely represent small scale structural changes and rearrangements in and around the bilin cofactor. The final reaction step takes place beyond the time-range of the IR measurements, although a comparison of the final time-resolved difference spectrum with a steady-state difference spectrum of the $P_{O}$ to $P_{F R}$ conversion (Figure $3 A$ ) shows that further spectral evolution in the IR region occurs prior to the final $P_{F R}$ formation. Hence, there are almost certainly some structural changes and rearrangement of the bilin environment beyond the $400 \mu \mathrm{s}$ of these measurements.

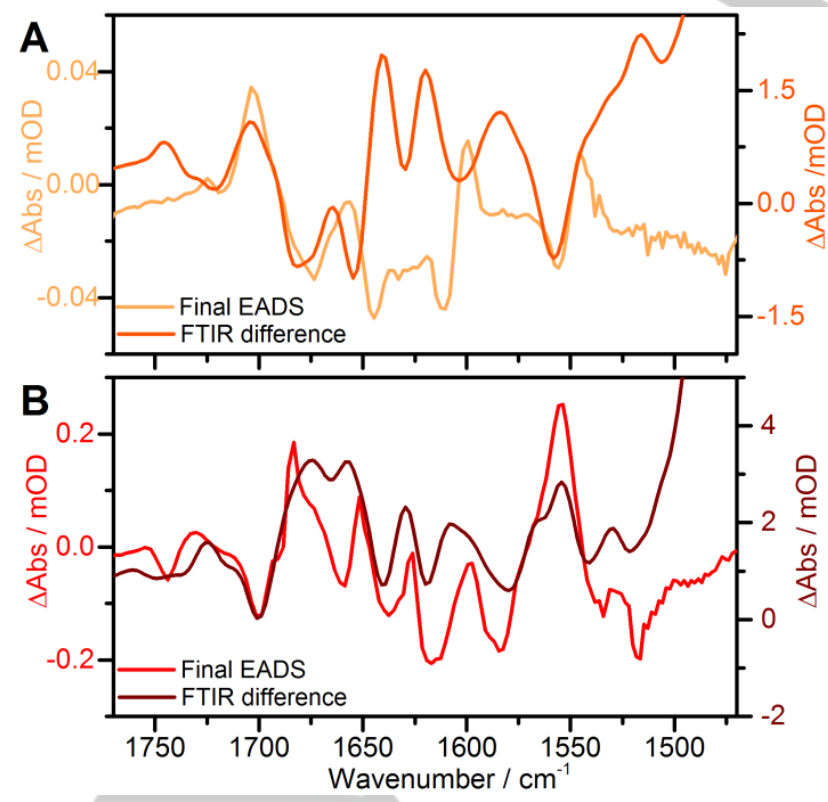

Figure 3. Comparison of the final EADS from global analysis of the timeresolved IR measurements (light coloured line, left axis) with the static infra- red difference spectra (dark coloured line, right axis) for $\mathbf{A}$. the $\mathrm{P}_{\mathrm{O}}$ to $\mathrm{P}_{\mathrm{FR}}$ and $\mathrm{B}$ the $\mathrm{P}_{\mathrm{FR}}$ to $\mathrm{Po}_{\mathrm{O}}$ photoconversions.

The absorbance feature with a maximum at $725 \mathrm{~nm}$ (EADS4), assigned to the final $P_{F R}$ state, is then formed with a lifetime of $57.1 \mathrm{~ms}$ (89.3 $\mathrm{ms}$ in $\mathrm{D}_{2} \mathrm{O}$ ). Whilst all reaction lifetimes except the first are slower in $\mathrm{D}_{2} \mathrm{O}$ compared to $\mathrm{H}_{2} \mathrm{O}$ the spectral shapes are virtually identical under both conditions. This implies that proton exchange does not affect the excited state properties or the isomerisation but may influence some of the slower steps that are likely to involve changes to the conformation of the bilin cofactor and the protein itself.

In the reverse reaction photoexcitation of the PFR state (Figure 4) results in quite different dynamics compared to the forward reaction, with five resolvable kinetic steps detected. In the visible region a fast, $0.3 \mathrm{ps}$ lifetime, component corresponds to the relaxation of a highly electronically excited state (e.g. $\mathrm{S}_{2}$ ) with intense ground state bleach and excited state absorption (EADS1) to a less electronically excited state (e.g. $\mathrm{S}_{1}$ ), the spectra of which retains some ground state bleach, but displays virtually no excited state features (EADS2). In the time region covered by both the visible and in the IR measurements three transitions are observed. EADS2 converts to EADS3 with a lifetime of 5-6 ps. In both the visible and IR regions the changes result in a significant loss of spectral intensity. In the visible region a very small positive feature is observed at around 670 $\mathrm{nm}$, and the intensity of the ground state bleach is again reduced. This is likely due to an intermediate with a very slightly blue-shifted absorption feature. In the IR region the transition corresponds to a change from an initially excited state to a spectrum with few spectral features. The feature arising from the $C=O$ of ring $D$ again provides a useful marker of the reaction progress and the strong bleach at $\sim 1700 \mathrm{~cm}^{-1}$ persists for the full $400 \mu \mathrm{s}$ IR timecourse. In EADS3 this bleach appears to be coupled to a transient feature at $\sim 1717 \mathrm{~cm}^{-1}$, a shift in the opposite direction to that observed in the $P_{0}$ to $P_{F R}$ reaction. We therefore assign this $5 \mathrm{ps}$ step to the photoisomerisation reaction. No spectral changes in either the visible or IR regions are then observed for several hundred nanoseconds. The transition from EADS3 to EADS4 occurs with a lifetime of $280 \mathrm{~ns}$ in the visible data in $\mathrm{H}_{2} \mathrm{O}$ (317 ns in $\mathrm{D}_{2} \mathrm{O}$ in the visible and $549 \mathrm{~ns}$ in the IR). In the visible region the intermediate absorption band shifts to $625 \mathrm{~nm}$ and in the IR a new bleach/transient feature at $1744 / 1730 \mathrm{~cm}^{-1}$ appears. Based on previous findings ${ }^{[19]}$ and the small kinetic isotope associated with this step it is likely that this feature is due to the deprotonation of one of the propionic side chains. The disappearance of the positive feature at $1717 \mathrm{~cm}^{-1}$ is assigned to the $\mathrm{C}=\mathrm{O}$ of ring $\mathrm{D}$, in keeping with previous studies of similar systems. ${ }^{[20]}$. The $625 \mathrm{~nm}$ absorbing intermediate described by EADS4 is then converted to an intermediate with an absorption maximum at $610 \mathrm{~nm}$ (EADS5) with a lifetime of $275 \mu \mathrm{s}\left(482 \mu \mathrm{s}\right.$ in $\left.\mathrm{D}_{2} \mathrm{O}\right)$. Due to the kinetic isotope effect associated with this step it is likely to involve reprotonation of the bilin factor. The IR data were only collected up to $400 \mu \mathrm{s}$, which is close to the lifetime found in the visible datasets for this step. Consequently, it is unlikely that this step will be kinetically well resolved in the IR region, hence the $96 \mu$ s lifetime.

For internal use, please do not delete. Submitted_Manuscript 
A

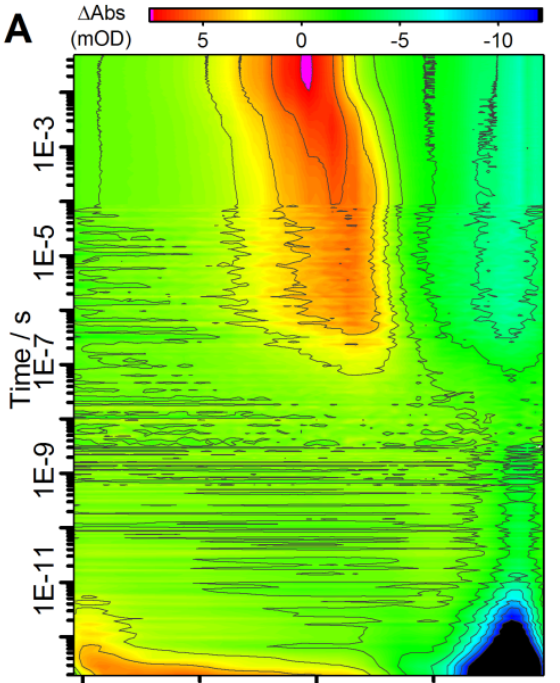

B

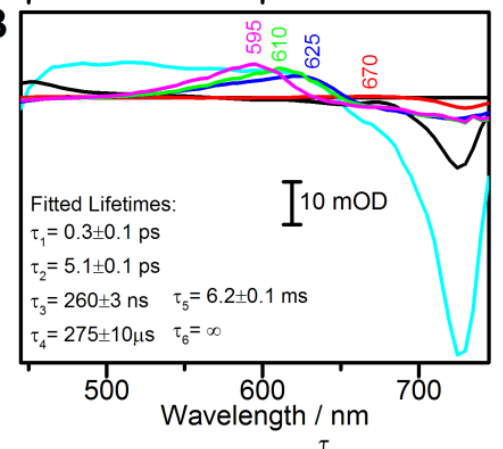

C

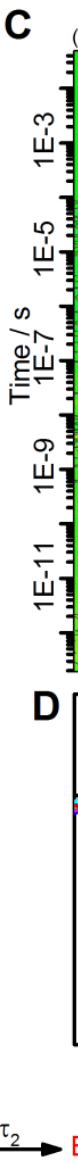

$\triangle$ Abs
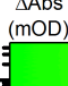

$-10$

$-5$

$10 \quad 5 \quad 0$
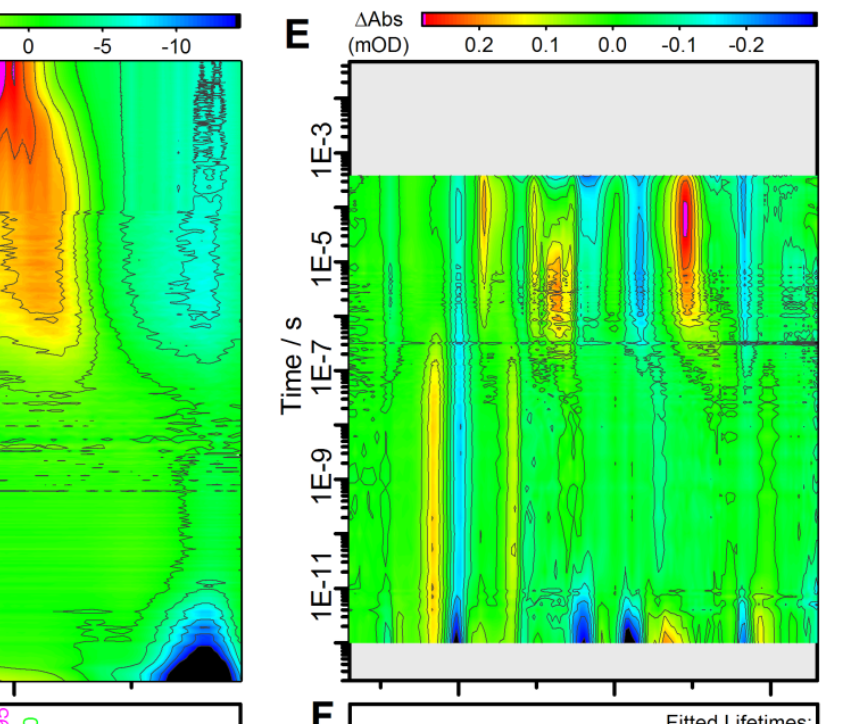

F

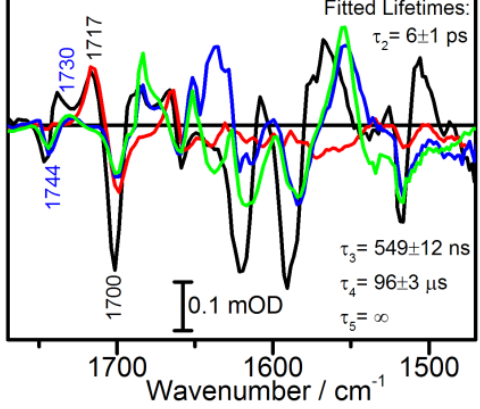

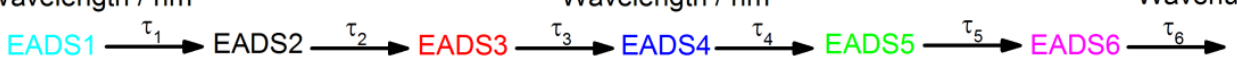

Figure 4. Contour maps of the raw data and evolution associated difference spectra (EADS) resulting from a global analysis of visible and infra-red transient absorption data, collected after excitation of the $\mathrm{P}_{\mathrm{FR}}$ state. $\mathbf{A}$ and $\mathbf{B}$, time-resolved visible data collected on Dten $\mathrm{Phy}$ in $\mathrm{H}_{2} \mathrm{O}$ based solution; $\mathbf{C}$ and $\mathbf{D}$, timeresolved visible data collected on Dten Phy in $\mathrm{D}_{2} \mathrm{O}$ based solution; . $\mathrm{E}$ and $\mathrm{F}$, time-resolved IR data collected on Dten Phy in $\mathrm{D}_{2} \mathrm{O}$ based solution. Visible data were recorded from $0.2 \mathrm{ps}$ until no further spectral changes were observed $(22.5 \mathrm{~ms})$, IR data were recorded over a 1 ps $-390 \mu \mathrm{s}$ window

A comparison of the final component from the global analysis of the time-resolved IR data with the steady state difference spectra (Figure $3 \mathrm{~B}$ ) reveals that whilst there are many similarities between the spectra there are still a number of spectral features that evolve after $400 \mu \mathrm{s}$. The majority of these changes occur $<1650 \mathrm{~cm}^{-1}$, which is in the region where protein absorption is expected to make significant contributions. Hence, the slower steps are only likely to involve large protein conformational changes and not significant changes in the bilin environment. The final step resolved in the visible dataset show the formation of the final $595 \mathrm{~nm}$ absorbing Po state (EADS6) with a lifetime of $6.2 \mathrm{~ms}\left(7.8 \mathrm{~ms}\right.$ in $\left.\mathrm{D}_{2} \mathrm{O}\right)$.

Studies of Phy systems with the well-studied red / far-red transitions suggest that in one direction the reaction consists of a picosecond isomerisation of the bilin cofactor. This is then followed by single bond rotations leading to the breakage and subsequent reformation of some of the $\mathrm{H}$-bond interactions with the protein environment. The other reaction direction also involves a picosecond isomerisation step, but this is then followed by deprotonation of the bilin chromophore, other minor structural changes, and then reprotonation to form the final stable state. ${ }^{[21]}$ It appears that despite the differences in wavelength sensitivity of Dten Phy a similar set of reactions occurs here. It is also known that the red / far-red Phys are capable of undergoing a much slower dark reversion process, which can occur over minutes or days. ${ }^{[22-24]}$ Therefore, measurements over the course of several hours were undertaken to identify any dark reactions from either of the two states of Dten Phy (Figures S7-8). The PFR state fully converts to the Po state within a few hours (lifetime of around 2 hours) in the dark. However, the Po state does not undergo any significant dark reversion back to the PFR state over a 24 hour time period. In typical red / far-red Phys it is known that the slower step(s), which follow the initial photoisomerisation, involve a series of large-scale conformational changes or domain motions in the protein. $^{[10,11,25,26]}$ We have used time-resolved solution X-ray scattering measurements to characterise the nature and kinetics of the structural dynamics during the $P_{O}$ to $P_{F R}$ transition in Dten Phy. A large difference signal in the small/wide angle region $(q<$ $0.25 \AA^{-1}$ ) was observed between the two states, indicating significant structural changes on the nanometre scale. The difference signal develops in both intensity and shape on the $\mu \mathrm{s}$ to second timescale (Figure 5A).

For internal use, please do not delete. Submitted_Manuscript 


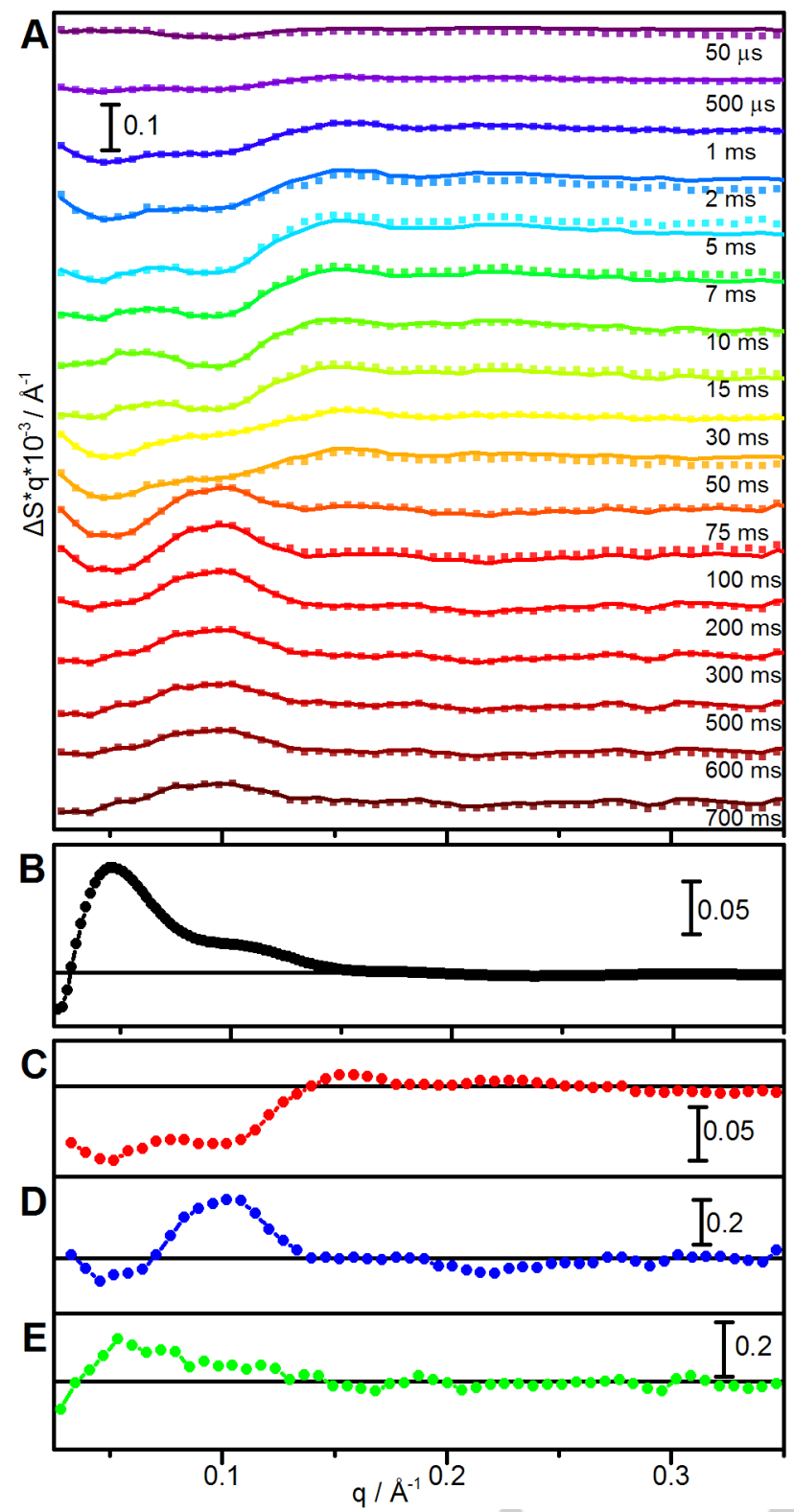

Figure 5. A. Laser-induced time-resolved X-ray scattering difference patterns for Dten Phy in solution, vertically offset for clarity, points are experimental data, lines are linear combinations of the first three basis patterns obtained by singular value decomposition. Note that scattering differences are multiplied by the scattering vector $q$ to reduce differences in the amplitude scale in SAXS and WAXS regions. B. Static X-ray scattering difference between $P_{O}$ and $P_{F R}$ states and C, D, E. the three main basis patterns obtained by SVD analysis of the entire time resolved $\mathrm{X}$-ray scattering dataset. opening motion that has been previously described for the bacterial red / far-red Phy family. ${ }^{[1,27]}$ The third basis pattern is the signal of the final state, as revealed by the comparison with a static difference between $P_{F R}$ and $P_{O}$ stationary states (Figure $5 B)$. A kinetic analysis of the whole data set in terms of the three main basis patterns reveals that the structural dynamics of Dten Phy evolve with three time constants of $\tau_{1}=8.7 \mathrm{~ms}, \tau_{3}=60 \mathrm{~ms}$ and $\tau_{3}>500 \mathrm{~ms}$. The second of these time constants correlates well with the $57.1 \mathrm{~ms}$ lifetime from the visible time-resolved data. Previous measurements have proposed that the X-ray scattering signal obtained for bacterial Phys represents an opening of the homodimer in the $\mathrm{PFR}_{\mathrm{FR}}$ state, caused by an increase in the separation of the opposing PHY domains. ${ }^{[11,27]}$ The similar signal obtained for Dten Phy in the second basis pattern suggests that this motion is conserved in algal Phys on the ms timescale. However, additional structural dynamics are observed for Dten Phy on the $\mu$ s timescale, which do not appear to be present in the bacterial Phys. Moreover, as further structural changes associated with $\mathrm{PO}_{\mathrm{O}}$ to $\mathrm{P}_{\mathrm{FR}}$ transition occur on slower timescales than the optical changes it is likely that these final conformational changes in the protein do not significantly affect the environment of the bilin chromophore.

The presence of these large scale structural changes in the protein have also been confirmed by viscosity-dependence studies. Visible time-resolved experiments at a range of solvent viscosities $(0-60 \%$ glycerol) revealed that the rates of the slower $\mu \mathrm{s}-\mathrm{ms}$ steps are strongly dependent on the solvent viscosity (Figure 6). The lifetime of the final step observed in the $P_{0}$ reaction increases 44 -fold in $60 \%$ glycerol, and the $P_{F R}$ final lifetime 8-fold. This strongly indicates that these slower steps in the photocycle involve a series of solvent-coupled protein motions. Conversely, no such dependence on solvent viscosity could be observed on the sub-ns dynamics (Figure S9).
A singular value decomposition analysis revealed that all the structural data can be described by three main time-independent basis patterns (Figure $5 \mathrm{C}-\mathrm{E}$ ), which form a time-independent orthonormal basis on which the full set of time-dependent data can be decomposed. The first, which represents the shape of the signal developing on the $\mu$ s to ms timescale, shows multiple structural changes across all regions. The second basis pattern describes the signal developing in the tens of ms and contains an oscillation that is similar to the signature of the quaternary

For internal use, please do not delete. Submitted_Manuscript 

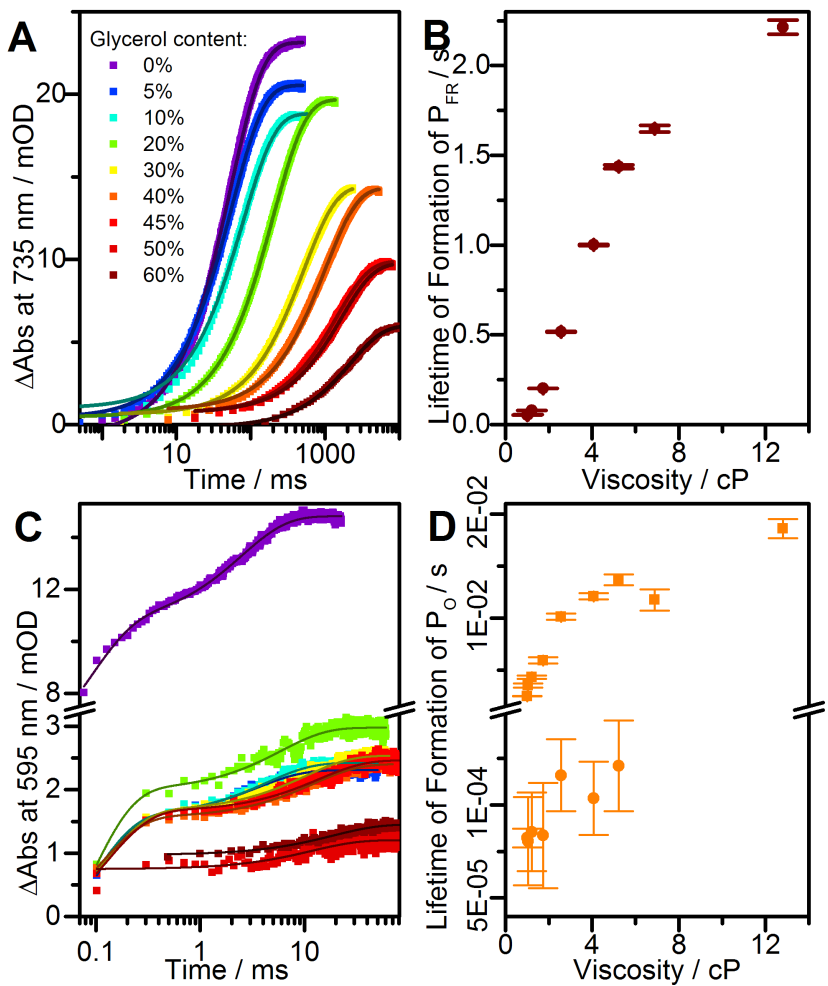

Figure 6. A. and C. The formation of $P_{F R}$ and $P_{O}$ states, following photoexcitation of $\mathrm{PO}_{\mathrm{O}}$ and $\mathrm{PFR}$ states respectively at a range of glycerol concentrations. Data (points) were fitted to a single or double exponential function (lines). B and D The resulting lifetimes, with fitting errors, are shown as a function of viscosity.

\section{Conclusions}

Taken together, the time-resolved approaches described here have allowed us to investigate the entire photocycle of an atypical algal Phy that is sensitive to orange / far-red light (Fig. 7). It is obvious that the photochemical mechanism of Dten Phy is much more complex than initially observed from time-resolved visible spectroscopy measurements. It has only been possible to unravel these hidden complexities by using additional IR and Xray scattering probes to study the initial photoisomerisation and slower, structural changes in the protein, respectively. Similar to the red / far-red photocycle in typical Phys it appears as though the orange / far-red photocycle of Dten Phy involves an initial photoisomerisation around the $\mathrm{C} 15-\mathrm{C} 16$ double bond between ring $C$ and $D$ of the bilin on the ps timescale. Following photoisomerisation, the formation of the final $\mathrm{P}_{\mathrm{FR}}$ or $\mathrm{Po}_{\mathrm{O}}$ state involves multiple small and large-scale structural changes in the protein, not all of which are linked to spectral changes associated with the bilin cofactor. Time-resolved X-ray scattering measurements have identified 3 kinetically distinct structural transitions, one of which is similar to opening of the dimer interface that has been observed previously in bacterial Phys. ${ }^{[11}$, 27] This study illustrates how crucial it is to use multiple biophysical tools in order to obtain a complete mechanistic understanding of these complex light-activated proteins.
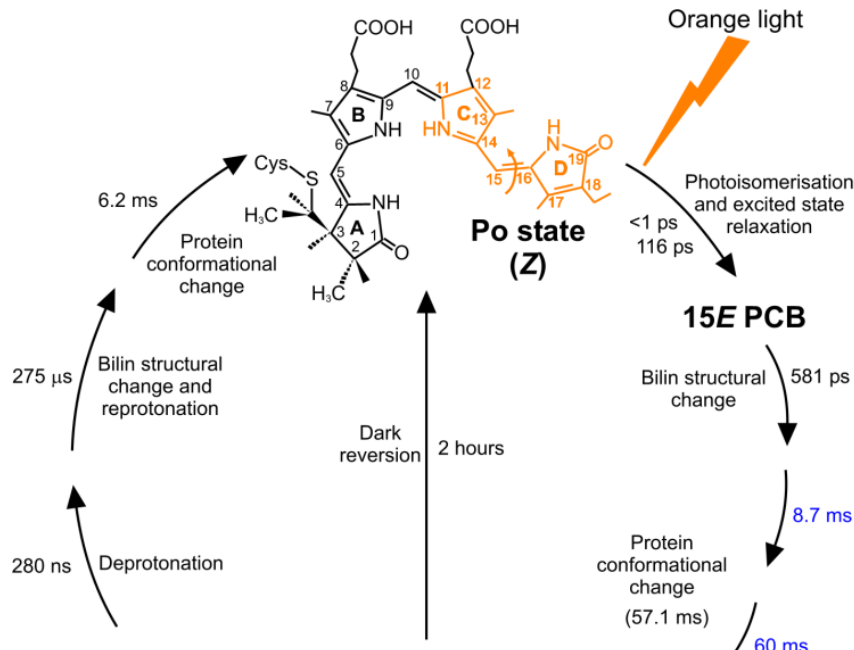

(Z)

15E PCB
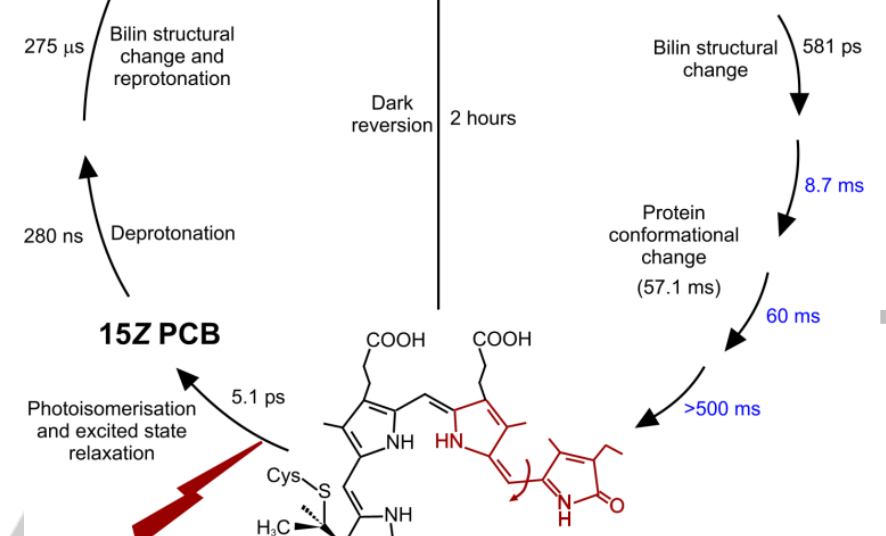

Far-red light<smiles>CC12CCCC1(C)C(C)(C)/C(=N\O)NC2=O</smiles>

(E)

Figure 7. Schematic overview of Dten Phy photocycle. Lifetimes derived from visible transient absorption ( $p s-m s$ ) in $\mathrm{H}_{2} \mathrm{O}$ are shown in black and the protein structural changes identified from S/WAXS (ms-s) are shown in blue.

\section{Experimental Section}

Sample Preparation and Basic Characterisation: The Dolihomastix tenuilepis gene encoding the photosensory regions PAS-GAF and PHY was ordered from GeneArt, subcloned into pET21a. The vector was then co-transformed along with pCola Duet vector containing pcyA and $\mathrm{HO} 1$ genes into HMS 174 cells. Cultures were grown at $30^{\circ} \mathrm{C}, 190 \mathrm{rpm}$ until an OD of 0.1 at $600 \mathrm{~nm}$ was achieved. The temperature was then dropped to $25^{\circ} \mathrm{C}$. Cultures were induced using $100 \mu \mathrm{M}$ IPTG at an OD of between 0.4 and 0.6 at $600 \mathrm{~nm}$, and grown overnight. Nickel-IDA resin was used as the first step purification. The resin was equilibrated with 5 column volumes of 'buffer A', $50 \mathrm{mM}$ Tris $\mathrm{pH} 7.5,300 \mathrm{mM} \mathrm{NaCl}, 5 \mathrm{mM}$ imidazole, $7.5 \%$ glycerol, $1 \% \beta$-mercaptoethanol, and incubated with the protein for 3 to 4 hours. The protein flow through was collected and the resin washed with 4 column volumes of 'buffer B', $50 \mathrm{mM}$ Tris $\mathrm{pH} 7.5$ $300 \mathrm{mM} \mathrm{NaCl}, 30 \mathrm{mM}$ imidazole, $7.5 \%$ glycerol, $1 \%$-mercaptoethanol. The protein was eluted with 'buffer C', $50 \mathrm{mM}$ Tris $\mathrm{pH} 7.5,300 \mathrm{mM} \mathrm{NaCl}$, $250 \mathrm{mM}$ imidazole, 7.5\% glycerol, $1 \%$-mercaptoethanol. Size exclusion was carried out on ÄKTA Pure (GE Healthcare) using a $\mathrm{Hi}$ Load 26/60 200 (GE Healthcare) column. The gel filtration column was equilibrated with two column volumes of gel filtration equilibration 'buffer D', $50 \mathrm{mM}$ Tris $\mathrm{pH}$ 7.5, $300 \mathrm{mM} \mathrm{NaCl}, 7.5 \%$ glycerol, $1 \% \beta$ mercaptoethanol before the protein was injected. After injection, the protein was eluted in two-column volume gel filtration equilibration buffer. The relevant fractions were analyzed using SDS-PAGE to pool together the fractions containing the purest protein. The protein was then concentrated using a Sartorius Stedim Biotech vivaspin column with a 30 $\mathrm{kDa}$ cut-off, flash-frozen and stored at $-80{ }^{\circ} \mathrm{C}$. UV/Vis absorption spectroscopy was performed using a Cary 50 UV-Vis Spectrophotometer, Varian, Agilent Technologies. All spectra were recorded from 300 to 800 $\mathrm{nm}$. Thorlabs mounted high power LEDs (of either 530 or $735 \mathrm{~nm}$ ) were

For internal use, please do not delete. Submitted_Manuscript 
used in the dark to convert the phytochromes into their different isomeric states. For studies of the dark thermal conversion over time, the cuvette was left inside a fully covered spectrometer. Steady state difference spectra in the infra-red region were collected using a Brucker Vertex 80 FTIR, measurements were recorded on samples $(\sim 100 \mu \mathrm{M})$ in $\mathrm{D}_{2} \mathrm{O}$ buffer (50 mM Tris pD 7.5, $300 \mathrm{mM} \mathrm{NaCl}, 7.5 \%$ glycerol, $1 \% \quad \beta$ mercaptoethanol) contained between $\mathrm{CaF}_{2}$ windows separated by a 100 $\mu \mathrm{m}$ Teflon spacer. Difference spectra were recorded for each state ( $P_{0}$ or $\mathrm{P}_{\mathrm{FR}}$ ) using the opposite state ( $\mathrm{P}_{\mathrm{FR}}$ or $\mathrm{P}_{\mathrm{O}}$ respectively) as the background measurement. For the viscosity studies $200 \mu \mathrm{l}$ of $10 \mathrm{mg} / \mathrm{ml}$ protein was added $800 \mu$ of $5,10,20,30,40,45,50$ and 60 per cent glycerol. Data from the photoconversion measurements were used as the $0 \%$ glycerol sample. Data were fitted with a single exponential ( $\mathrm{P}_{\mathrm{FR}}$ formation and $\mathrm{Po}_{\mathrm{O}}$ formation at 50 and $60 \%$ glycerol) or double exponential (Po formation $<45 \%$ glycerol) function

Nanosecond laser flash photolysis: The phytochrome photodynamics were studied using a laser system consisting of an optical parameter oscillator (OPO) pumped by a Q-switched Nd: YAG laser (Brilliant B Quantel). Excitation wavelengths were generated using the OPO pumped by the third harmonic of the laser. A quartz cuvette with a $2 \mathrm{~mm}$ path length for pump-excitation and $10 \mathrm{~mm}$ path length for probing of the was used. Samples had an O.D. of 0.8 at $735 \mathrm{~nm}$ in the $10 \mathrm{~mm}$ path. TA spectra were recorded using an LKS-60 Flash-Photolysis instrument (Applied Photophysics Ltd.). Kinetics were recorded in $5 \mathrm{~nm}$ increments across the visible spectral region. Samples illuminated with a $530 \mathrm{~nm}$ LED to generate the $P_{F R}$ state were then photoexcited at $430 \mathrm{~nm}$ with 15 $\mathrm{mJ}$ pulse energy (this excitation wavelength was selected due to the limited pump wavelength range of the laser system) kinetics were recorded over a timescale of $0.05-47.5 \mathrm{~ms}$. Samples were illuminated with a $735 \mathrm{~nm}$ LED to generate the Po state were then photoexcited at $595 \mathrm{~nm}$ with $9 \mathrm{~mJ}$ pulse energy, kinetics were recorded over a timescale of $0.1-495 \mathrm{~ms}$.

Ultrafast spectroscopy: Time-resolved visible spectroscopy was performed using a Ti:sapphire amplifier system (Newport Spectra Physics, Solstice Ace) producing $800 \mathrm{~nm}$ pulses at $1 \mathrm{kHz}$ with $100 \mathrm{fs}$ pulse duration was used for ultrafast pump-probe studies. A TOPAS Prime optical parameter amplifier (OPA) with associated NirUVis unit was pumped with a portion of the output of the amplifier to generate the pump beams. A broadband ultrafast pump-probe transient absorbance spectrometer 'Helios' (Ultrafast Systems LLC) was used to collect data over a $3 \mathrm{~ns}$ time window with a time resolution of around $250 \mathrm{fs}$. The probe beam consisted of a white light continuum generated in a $\mathrm{CaF}_{2}$ crystal and absorbance changes were monitored between 390 and 745 $\mathrm{nm}$. A broadband sub-nanosecond pump-probe transient absorption spectrometer 'Eos' (Ultrafast Systems LLC) was used to collect data on longer timescales. A $2 \mathrm{kHz}$ white-light continuum fiber laser was used to generate the probe pulses, the delay between pump and probe was controlled electronically. Samples reservoirs were illuminated with 530 or $735 \mathrm{~nm}$ LEDs and flowed through a $0.2 \mathrm{~mm}$ path length quartz cuvette. Samples had an O.D. of around 0.5 at $735 \mathrm{~nm}$ in the $0.2 \mathrm{~mm}$ path Excitation wavelengths of $430 \mathrm{~nm}$ and $595 \mathrm{~nm}$, with $0.5 \mathrm{uJ}$ pulse energy, were used over timescales up to $82 \mu \mathrm{s}$. The pre-excitation data were subtracted before the analysis and spectral chirp corrected for.

Time-resolve infra-red spectroscopy was performed using the TRMPS set-up of the ULTRA-LIFEtime system ${ }^{[28]}$ at the Central Laser Facility, STFC, Rutherford Appleton Laboratory, UK. This uses a $100 \mathrm{kHz}$ ultrafast laser based on a custom dual $\mathrm{Yb}: \mathrm{KGW}$ system (Pharos, Light Conversion). Samples in $\mathrm{D}_{2} \mathrm{O}$ buffer (50 mM Tris pD 7.5, $300 \mathrm{mM} \mathrm{NaCl}$, $7.5 \%$ glycerol, $1 \% \beta$-mercaptoethanol) were contained between two $\mathrm{CaF}_{2}$ windows, separated by a teflon spacer to give a pathlength of approximately $50 \mu \mathrm{m}$. Samples reservoirs were illuminated with 530 or
$735 \mathrm{~nm}$ LEDs and flowed through the cell, the sample holder was rastered to avoid sample damage. Excitation wavelengths of $575 \mathrm{~nm}$ at 1 $\mathrm{kHz}$ repetition rate and $0.6 \mu \mathrm{J}$ pump energy, and $735 \mathrm{~nm}$ at $500 \mathrm{~Hz}$ repetition rate and $2 \mu \mathrm{J}$ pump energy, were used. Data were collected for approximately 35 and 100 minutes respectively per dataset The excitation beam was set at the magic angle with respect to the IR probe beam. Difference spectra were generated relative to the ground state in the spectral window $1470-1770 \mathrm{~cm}^{-1}$ at time delays ranging between 1 ps and $390 \mu \mathrm{s}$. Pixel to wavenumber calibration was performed using a polystyrene standard. Infra-red pump-probe transient absorption data from the TRMPS set-up were averaged $0.1 \mu$ s on either side of each 10 $\mu \mathrm{s}$ "TRMP" step after the first $10 \mu$ s of data. Thus 105 ( $P_{\mathrm{FR}}$ reaction) or 57 (Po reaction) time points were spread over the first $10 \mu$ s timerange, and thereafter timepoints were every $10 \mu \mathrm{s}$ up to $390 \mu \mathrm{s}$ (39 in total)

Global Analysis: Global Analysis was performed using the open-source software Glotaran to obtain an evolution associated difference spectra $(E A D S)^{[29]}$. This procedure reduces the matrix of change in absorbance as a function of time and wavelength, to a model of one or more exponentially decaying time components, each with a corresponding difference spectrum (EADS). All datasets were fitted to a sequential, unbranched model. Errors quoted with the lifetime values are the standard errors calculated during the global analysis. Visible spectrum data sets from the nanosecond laser flash photolysis and ultrafast transient absorption techniques were combined into one dataset. The wavelength points from the ultrafast data were removed so that the data matched the those of the laser flash photolysis data ( $5 \mathrm{~nm}$ steps). The datasets were then scaled to match intensities of the major spectral features in overlapping time regions.

Time Resolved Small and Wide Angle X-ray Scattering: TR-S/WAXS experiments have been performed at the beamline ID09 of the European Synchrotron Radiation facility. A sample of Dten Phy $(250 \mu \mathrm{M})$ was continuously illuminated by a $730 \mathrm{~nm}$ LED to stabilise the Po form, flowed by a peristaltic pump (Gilson Minipulse 3) into a $2 \mathrm{~mm}$ quartz capillary and excited by a $5 \mathrm{~ns}$ laser pulse (EKSPLA) at $595 \mathrm{~nm}\left(1 \mathrm{~mJ} / \mathrm{mm}^{2}\right)$. The $X$-ray scattering signal was monitored at different time delays after laser excitation by $20 \mu \mathrm{s} X$-ray pulse train. Scattering patterns were normalised the water peak (about $2.2 \AA^{-1}$ ) before making differences between laseron and laser-off patterns. The time evolution of the solvent heating peak at $1.8 \AA^{-1}$ was checked, and did not affect the scattering patterns in the $q$ range where protein difference signal used for analysis was present (0$0.3 \AA^{-1}$ ). Singular value decomposition analysis of time resolved scattering dataset was performed using an in-house developed Python based code. Visual inspection and autocorrelation analysis of both basis patterns and amplitude vectors ${ }^{[30]}$ indicated the first three basis patterns containing significant information, with the rest containing essentially only random noise. A linear combination of the first three basis patterns was then used to fit the time evolution of the scattering signal and to extract time constants.

\section{Acknowledgements}

Time-resolved visible absorption measurements were performed at the Ultrafast Biophysics Facility, Manchester Institute of Biotechnology, as funded by BBSRC Alert14 Award $B B / M 011658 / 1$. Time resolved infrared measurements were carried out through program access support of the UK Science and Technology Facilities Council (STFC). Studentship funding (UC) from Newport is gratefully acknowledged.

For internal use, please do not delete. Submitted_Manuscript 
Keywords: Photochemistry • Biophysics • Photoreceptor • Phytochrome - Time-resolved spectroscopy

[1] N. C. Rockwell, Y.-S. Su, Lagarias, Annu. Rev. Plant Biol. 2006, 57, 837-858.

[2] N. C. Rockwell, Lagarias, Chem. Eur. J. of Chem. Phys. 2010, 11 1172-1180.

[3] F. Andel, Lagarias, R. A. Mathies, Biochemistry 1996, 35, 1599716008.

[4] H. Foerstendorf, T. Lamparter, J. Hughes, W. Gärtner, F. Siebert, Photochem. Photobiol. 2000, 71, 655-661.

[5] A. Remberg, I. Lindner, T. Lamparter, J. Hughes, C. Kneip, P. Hildebrandt, S. E. Braslavsky, W. Gärtner, K. Schaffner, Biochemistry 1997, 36, 13389-13395.

[6] C. Kneip, P. Hildebrandt, W. Schlamann, S. E. Braslavsky, F. Mark, K. Schaffner, Biochemistry 1999, 38, 15185-15192.

[7] W. Rüdiger, F. Thümmler, E. Cmiel, S. Schneider, Proc. Natl. Acad. Sci. U.S.A. 1983, 80, 6244-6248.

[8] C. Schumann, R. Gross, M. M. N. Wolf, R. Diller, N. Michael, T. Lamparter, Biophys. J. 2008, 94, 3189-3197.

[9] P. Schmidt, T. Gertsch, A. Remberg, W. Gärtner, S. E. Braslavsky, K. Schaffner, Photochem. Photobiol. 1998, 68, 754-761.

[10] E. S. Burgie, T. Wang, A. N. Bussell, J. M. Walker, H. Li, R. D. Vierstra, J. Biol. Chem. 2014, 289, 24573-24587.

[11] H. Takala, A. Björling, O. Berntsson, H. Lehtivuori, S. Niebling, M. Hoernke, I. Kosheleva, R. Henning, A. Menzel, J. A. Ihalainen, et al., Nature 2014, 509, 245-248.

[12] J. J. Tabor, A. Levskaya, C. A. Voigt, J. of Mol. Biol. 2011, 405, 315324.

[13] S. R. Schmidl, R. U. Sheth, A. Wu, J. J. Tabor, ACS Synth Biol 2014, 3, 820-831.

[14] N. C. Rockwell, D. Duanmu, S. S. Martin, C. Bachy, D. C. Price, D. Bhattacharya, A. Z. Worden, Lagarias, Proc. Natl. Acad. Sci. U.S.A. 2014, 111, 3871-3876

[15 ] A. F. E. Hauck, S. J. O. Hardman, R. J. Kutta, G. M. Greetham, D. J. Heyes, N. S. Scrutton, J. Biol. Chem. 2014, 289, 17747-17757.
[16 ] S. J. O. Hardman, A. F. E. Hauck, I. P. Clark, D. J. Heyes, N. S. Scrutton, Biophys. J. 2014, 107, 2195-2203.

[17] A. Takiden, F. Velazquez-Escobar, J. Dragelj, A. L. Woelke, E.-W. Knapp, P. Piwowarski, F. Bart, P. Hildebrandt, M. A. Mroginski, Photochem. Photobiol. 2017, 93, 713-723.

[18] F. Velazquez Escobar, T. Utesch, R. Narikawa, M. Ikeuchi, M. A. Mroginski, W. Gärtner, P. Hildebrandt, Biochemistry 2013, 52, 48714880.

[19] F. Velazquez Escobar, P. Piwowarski, J. Salewski, N. Michael, M. Fernandez Lopez, A. Rupp, B. M. Qureshi, P. Scheerer, F. Bartl, N. Frankenberg-Dinkel, F. Siebert, M. Andrea Mroginski, P. Hildebrandt, Nat Chem 2015, 7, 423-430.

[20] T. Stensitzki, Y. Yang, A. L. Wölke, E.-W. Knapp, J. Hughes, M. A. Mroginski, K. Heyne, Photochem. Photobiol. 2017, 93, 703-712.

[21] C. Song, T. Rohmer, M. Tiersch, J. Zaanen, J. Hughes, J. Matysik, Photochem. Photobiol. 2013, 89, 259-273.

[22] E. Giraud, J. Fardoux, N. Fourrier, L. Hannibal, B. Genty, P. Bouyer, B. Dreyfus, A. Verméglio, Nature 2002, 417, 202-205.

[23] B. Karniol, R. D. Vierstra, Proceedings of the National Academy of Sciences 2003, 100, 2807-2812.

[24] R. Tasler, T. Moises, N. Frankenberg-Dinkel, FEBS J. 2005, 272, 1927-1936

[25] A. T. Ulijasz, G. Cornilescu, C. C. Cornilescu, J. Zhang, M. Rivera, J. L. Markley, R. D. Vierstra, Nature 2010, 463, 250-254.

[26] D. J. Heyes, B. Khara, M. Sakuma, S. J. O. Hardman, R. O'Cualain, S. E. J. Rigby, N. S. Scrutton, PLoS ONE 2012, 7, e52418.

[27] A. Bjorling, O. Berntsson, H. Lehtivuori, H. Takala, A. J. Hughes, M. Panman, M. Hoernke, S. Niebling, L. Henry, R. Henning, et al., Science Advances 2016, 2, e1600920-e1600920.

[28] G. M. Greetham, P. M. Donaldson, C. Nation, I. V. Sazanovich, I. P. Clark, D. J. Shaw, A. W. Parker, M. Towrie, Appl. Spectrosc. 2016, 70, 645-653.

[29] J. J. Snellenburg, S. P. Laptenok, R. Seger, K. M. Mullen, I. H. M. van Stokkum, J. Stat. Softw. 2012, 49, 1-22.

[30] E.R. Henry, J. Hofrichter, Meth. Enzymol. 1992, 210, 129-192.

\section{For internal use, please do not delete. Submitted_Manuscript}




\section{FULL PAPER}

Recently phytochromes have become attractive targets for optogenetic applications because of their ability to respond to multiple wavelengths of light. Here, we have used a combination of time-resolved visible, infra-red, and X-ray scattering techniques across picosecond to second timescales to map photochemical mechanisms and structural change in an atypical orange / far-red light sensitive algal phytochrome.

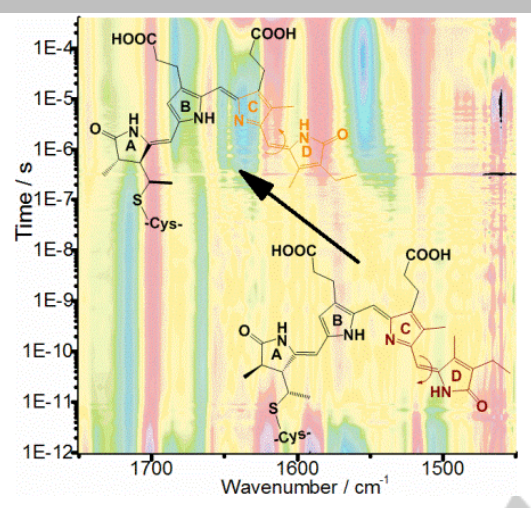

Uzma Choudry, Derren J. Heyes, Samantha J. O. Hardman, Michyo Sakuma, Igor V. Sazanovich, Joyce Woodhouse, Eugenio De La Mora, Martin Pedersen, Michael Wulff, Martin Weik, Giorgio Schirò, Nigel S. Scrutton*

Page No. - Page No.

Photochemical mechanism of an atypical algal phytochrome

For internal use, please do not delete. Submitted_Manuscript 